\title{
Progress in Bionanocomposites: From green plastics to biomedical applications
}

The term bionanocomposites was introduced several years ago, to define an emerging class of biohybrid materials, resulting from the combination of biopolymers such as polysaccharides, proteins, nucleic acids, etc. with inorganic solids at the nanometric scale [1] and [2]. Such inorganic fraction consists of finely divided solids, spanning from clays to phosphates or carbonates, whose origin can be either natural or synthetic. As in any multiphase material, the most significant challenge in bionanocomposites is to attain materials with superior performance, by the subtle management of the individual properties of its components. Notably, the biocompatible and biodegradable character of biopolymers, alongside with the mechanical and thermal properties of the inorganic counterpart, bridge the gap between functional and structural materials. In one word, it is all about synergy!

As will become increasingly clear for the reader throughout the collection of authoritative reviews in this special issue, the relevance of coupling biopolymers with inorganic nanomaterials, through innovative architectures is twofold. First, it contradicts the idea that materials are either eco-friendly or high-performance. Bionanocomposites constitute the demonstration that bioinspired design behind the synthesis of most bionanocomposites - allows combination of both the green characteristics and enhanced performances. Second, it demonstrates how the properties resulting from these biohybrid materials are of high significance in applications such as food packaging, gas-diffusion barrier, flame retardancy, sensing devices, drug delivery or regenerative medicine.

Contributions on processing of bionanocomposites, the use of diverse biopolymer sources like PLA and polysaccharides, the different building blocks used as inorganic counterpart, and applications of these biohybrid materials, constitute the backbone of this Special Issue.

We would like to acknowledge all the authors for their contributions to this collection of review articles. We believe that their collected efforts have resulted in an up-to-date selection of papers describing the latest developments in bionanocomposites and their societal impact. We are hence confident that the present contributions will provide the reader with a reference on state-of-the-art research in this topic.

We are deeply indebted to Professors Krzysztof Matyjaszewski and Guy C. Berry, Editors-in-Chief of Progress in Polymer Science, for giving us the opportunity to act as Guest Editors. Also we would like to thank the editorial staff at Elsevier Science Ltd., for their kind assistance in the management of this publication 